\title{
Skin Shrinkage with Vibration Amplification of Sound Energy at Resonance (VASER) Liposelection of Abdomen: Avoiding Abdominoplasty
}

\section{Dr Munir Alam}

\author{
MBBS (KE), FRCS (Ire), FRCS (Ed), FCPS (Plastic Surgery) \\ Int/European Board Certified Cosmetic Surgeon \\ Associate Professor of Plastic Surgery, Independent medical college, Faisalabad, Pakistan. \\ Consultant Plastic Surgeon, Faisal Hospital, Faisalabad, Pakistan
}

Corresponding Address: Dr Munir Alam; mrmuniralam@gmail.com

46 University Town, Millat Road, Faisalabad, Pakistan

Received 27 January 2020;

Accepted 16 February 2020;

Published 20 February 2020

\begin{abstract}
Objective / Purpose of the Study: For abdominal reshaping / contouring, patient with excess abdominal skin and redundant fat quite often require abdominoplasty / tummy tuck. Inconsistent results achieved with traditional liposuction especially in the supraumbilical area. This study is to assess the role of abdominal VASER liposelection and if the abdominoplasty can be avoided.

Material and Methods: This is retrospective cross sectional observational study conducted at Dundrum Medical Cosmetic Clinic, Dublin 16, Ireland. The study period includes two years from March 2009 to February 2011. In this study total number of consecutive 85 patients, operated by single surgeon were included with type I-IV patient category according to Matarasso classification system (1). There were 10 (11.8\%) male and $75(88.2 \%)$ female patient with mean age 42.4 years \pm 10.95 years.

Results: All of the 85 patients were treated with abdominal VASER liposelection. There were 75 female (88.2\%) patients and 10 male (11.8\%) patients in this study. Seven (8.2\%) female patient require second session of VASER liposelection procedure 3 months after the first procedure and $3(3.5 \%)$ female patient underwent third session to complete the abdominal contouring. None of the total 10 male patient required 2 nd or 3 rd session of abdominal VASER liposelection. The satisfaction level achieved 90 to $100 \%$ by using visual analogue scoring system for all types I IV abdomen. No early or late complication was noted in this study.

Conclusion: This is a low risk, minimally invasive, scarless ambulatory technique performed under local anaesthetic, highly satisfactory to the patient as well as surgeon and is effective in all the categories of protuberant abdomen avoiding the local and systemic risk of abdominoplasty.
\end{abstract}

Keywords: VASER Liposelection, abdominoplasty, tummy tuck, Liposuction, skin shrinkage

\section{Introduction}

The shape of the abdomen depends upon various factors i.e; bony skeleton, overlying muscles, the distribution of fat and intraabdominal contents. The frontal profile of abdomen extends superiorly from xiphisternum to pubic bones in the middle and inguinal ligament inferiorly. The flanks (love handle) extend laterally to variable degree.

According to Matarasso classification system there are four types of protuberant abdomen described ${ }^{[1]}$. Tradionally, Type I is the moderate amount of fat which can be treated with suction assisted lipectomy, Type II is the lower abdominal excess fat and skin laxity which can be treated with mini-abdominoplasty, Type III is the excess fat in the supra and infra-umbilical region with skin laxity which can be treated with modified abdominoplasty and the Type IV is the lax abdominal muscles \pm divarication of rectus abdominus muscles \pm excess fat supra and infra-umbilical region with abdominal wall skin laxity which require abdominoplasty and suction assisted lipectomy ${ }^{[2]}$.

The aesthetic and anatomic considerations are slightly different in males and female. The abdominal wall fat is well compartmentalized into three layers by Scarpa's and Camper's fascia. However, these anatomic distinctions to be of less significance than the deep, intermediate, and superficial surgical fat layers. The body contouring layers are based on the division of subcutaneous fat into thirds by depth and represent relative safety zones for liposuction treatment.

The deep layer is the one third of the fat that lies adjacent to the underlying muscle fascia. It is composed of loose, less compact fat that can be safely removed in most areas except the buttocks. The most commonly suctioned area is the intermediate layer of the 
abdominal fat (Figure 1). The body contour improved without altering the skin surface and with minimal risk of contour irregularities or thermal injury. The degree of skin contraction depends on the overall thickness of the excess fat layer, necessitating subsequent $2^{\text {nd }}$ or $3^{\text {rd }}$ session for complete elimination of the excess fat and shrinkage of skin laxity.

Figure 1: Three abdominal fat layers (VASERing and suction of abdominal fat 1A-1B, resulting in collapse of abdominal skin to muscle fascia 1C)

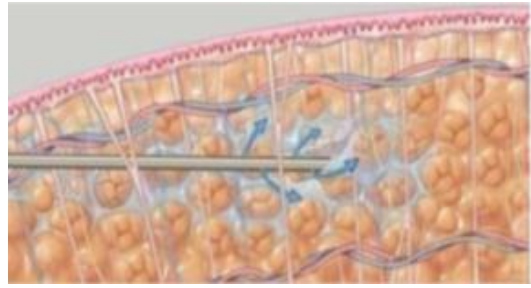

$\mathbf{1 A}$

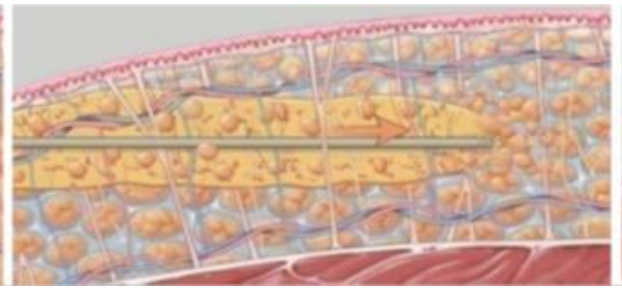

1B

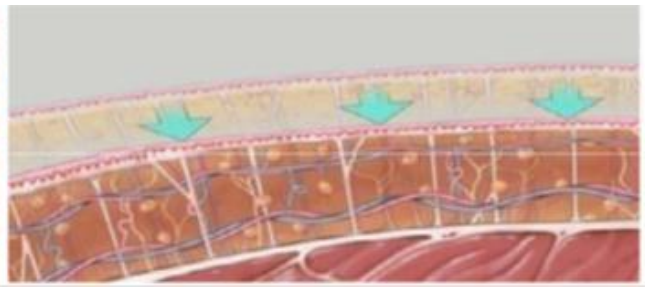

$1 \mathrm{C}$

Picture 1:

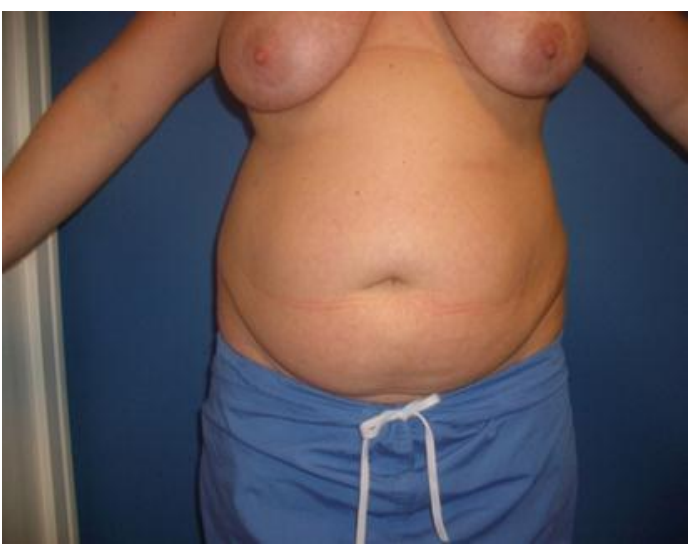

Picture 1 A: Pre Op

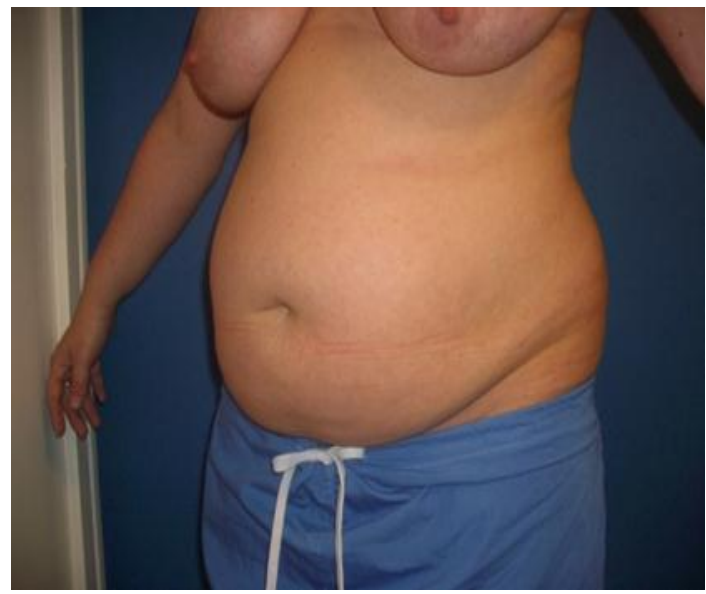

Picture 1 C: Pre Op

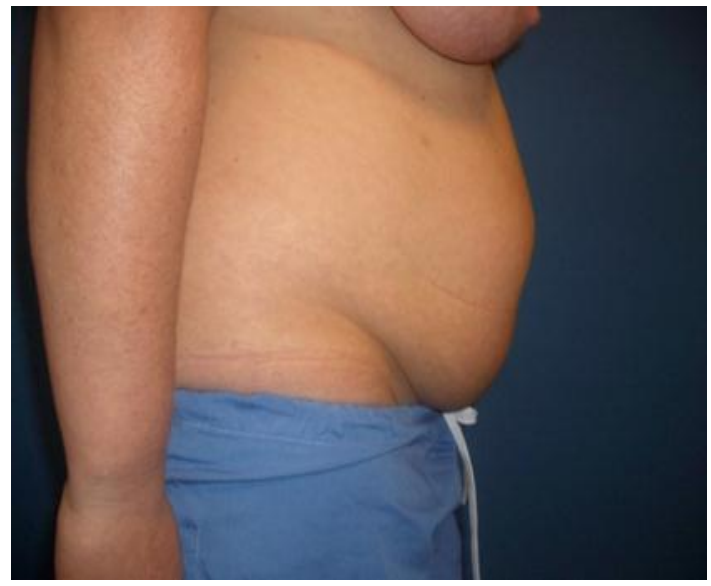

Picture 1 E: Pre Op

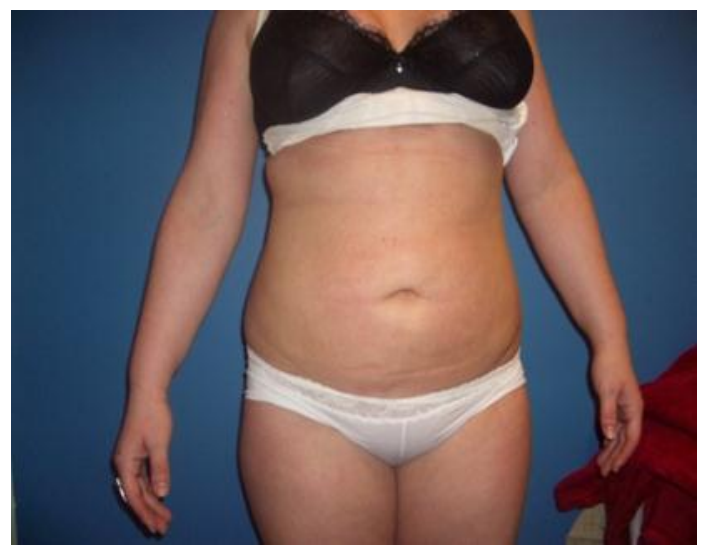

Picture 1 B: 3 months post op

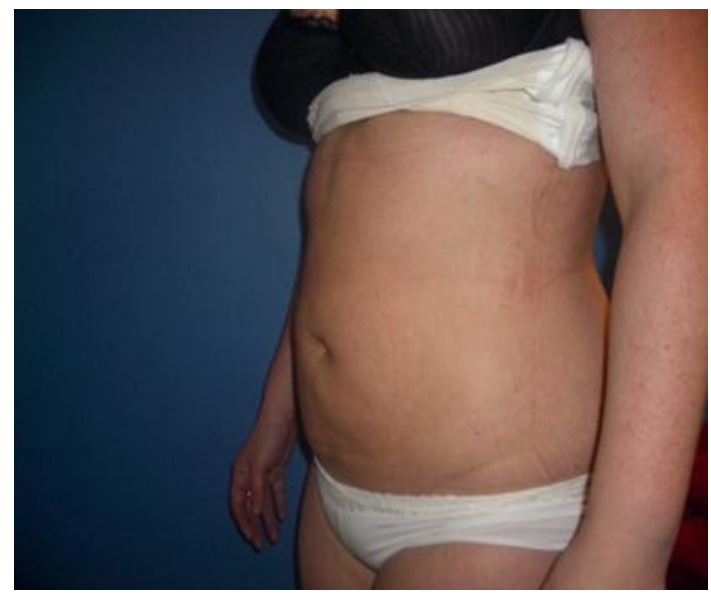

Picture 1 D: 3 months post op

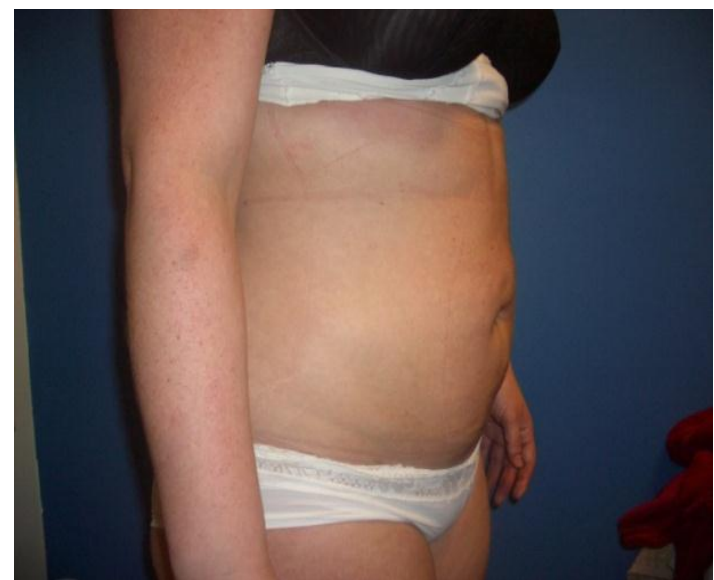

Picture 1 F: 3 months post op 


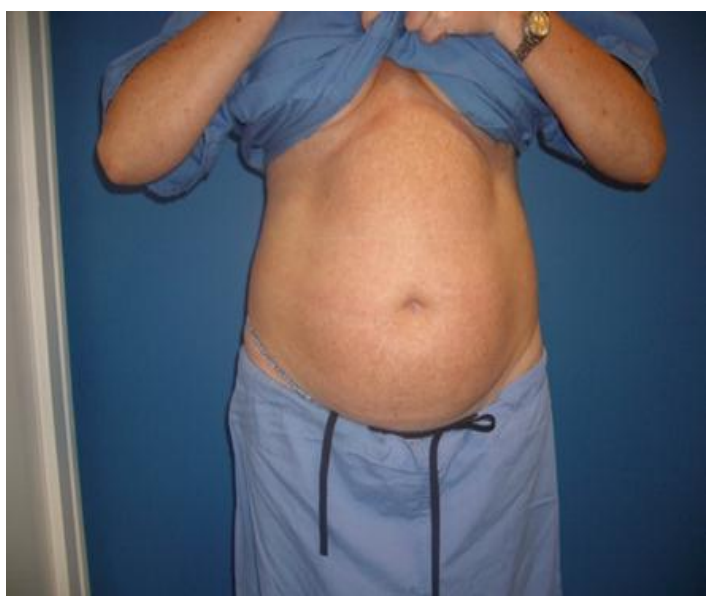

Picture 2 A: Pre Op

The superficial layer represents the upper one third of the subcutaneous fat and abuts the dermis. Body contouring of the superficial layer poses greater risks and should only be performed when there is enough experience in treating the deeper layers. In most instances the superficial fat layer is preserved during treatment of both males and females for smooth contouring of the abdominal wall surface.

Historically, the treatment of protuberant abdomen was major surgical procedure which often require multiple operations under general anesthesia with its risks like bleeding, hematoma/seroma, long scar and infections etc. The old-style liposuction was first used in early 1970s for type I or as a combination procedure with surgery ${ }^{[3,4,5]}$. The combined procedure using liposuction and abdominoplasty is known as lipoabdominoplasty ${ }^{[6,26]}$.

With the traditional surgical procedure, evolution of surgical procedures and advent of liposuction still require a procedure which can shrink the loose skin without the resection of lax abdominal skin i.e; procedure to avoid abdominoplasty for its attendant risks. Approximately $10 \%$ to $20 \%$ of patients suffer a local complication following abdominoplasty, while fewer than $1 \%$ suffer a systemic complication ${ }^{[6,26]}$.

Ultrasonic technology for isolating the adipocytes from the surrounding structures like blood vessels, nerves, collagen bundle, connective tissue etc was originated in Fance \& Italy during 1980s [7].

VASER liposelection is the most modern 4th generation of ultrasonic technology. In United States, it was modified from ultrasonic liposelection in 1998 into VASER Liposelection and was popularized all over the world. It is known as liposelection because vibration energy selectively isolate the adipocytes, sparing the surrounding structures.

\section{Material and Methods}

This is a retrospective cross sectional observational study of abdominal VASER liposelection performed by single surgeon at Dundrum Medical Cosmetic Clinic, Dundrum, Dublin 16, Ireland from March 2009 to February 2011. There were total of 85 patients in this study. There were $10(11.8 \%)$ male and 75 (88.2\%) female patients.

Inclusion criteria was all patients according to Matarasso classification system type I - IV, clinically fit for day case,

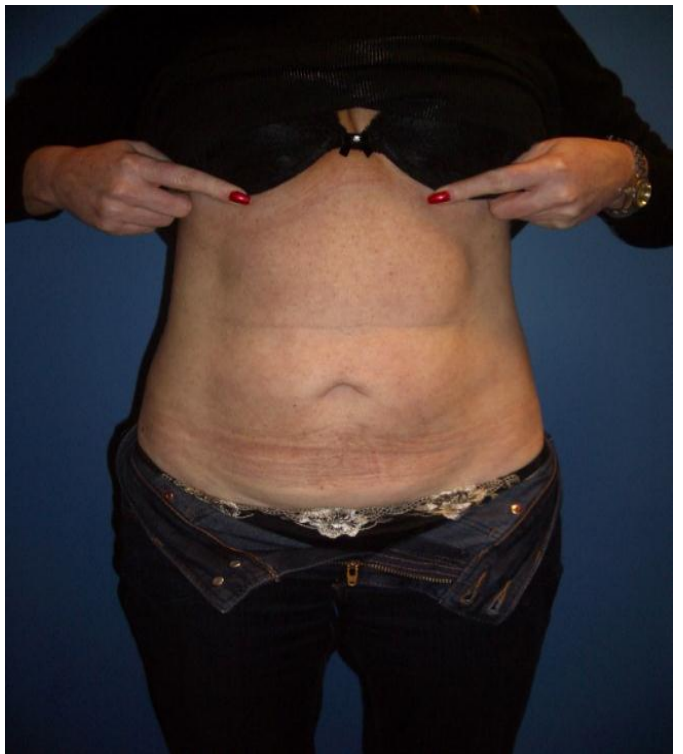

Picture 3 B: 6 months post op

ambulatory procedure. The detail medical and surgical history and thorough physical examination of previous abdominal operation, scar, hernia including body mass index (BMI) was observed.

Patients with co morbid disease, requiring admission in the hospital, pregnant, morbidly obese, diverication of recti or external abdominal hernia and psychologically unfit were excluded from the study.

The informed consent related to pre operative and post operative pictures, nature and limitation of the procedure, benefits and risks of surgery was obtained. All the patients who had 2nd or 3rd session of VASER liposelection were consented, explained about the procedure and subsequent sessions on a first consultation.

\section{Surgical Technique}

The patient is marked preoperatively in standing position. Then patient is placed supine position for supra and infra- umbilical region, left lateral for right flank and right lateral for left flank. Three stab access incision approximately 3 to $4 \mathrm{~mm}$ in size with number 11 blade for supra and infraumbilical area. One or two stab incision on the superior and/or inferior aspect for flank area. The local anesthetic is infiltrated into the abdominal fat as tumescent technique with the mixture of lidocaine $35 \mathrm{mg} / \mathrm{Kg}$ body weight and $1 \mathrm{ml}$ of adrenaline in $0.9 \%$ normal saline $(1000 \mathrm{ml})$ and Sodium bicarbonate $10 \mathrm{ml}$. Tumescent technique is a clinical term that the fluid is infiltrated into the tissue plane when the infused fat with overlying skin become tight. To define it exactly, pure tumescent liposuction technique is performed under local anesthetic which excludes the use of general anesthesia and intravenous sedation $[23,24,25]$.

After infiltration of fluid with tumescent technique, abdominal vasering of the adipocytes suspended in tumescent fluid for approximately 30 minutes and finally aspiration is performed which contain approximately $70 \%$ fat suspended in approximately $30 \%$ infiltrate fluid. The aspiration amount range from two to five liters average 3.5 liters.

VASER liposelection is a dynamic technique, and the VASER probe must be in motion at all times to prevent heat buildup and thermal injury.

All access sites are messaged to remove the remaining fluid. The access incisions are closed with steristrips after cleaning with tincture benzoin co and antiseptic waterproof dressing is applied to 
make it convenient showering the next day of the procedure. Manual lymphatic message is encouraged usually after 2-3 days post operatively twice a day for 20-30 minutes and customized compression garments worn most of the time especially night time for 2 to 3 months.

The patient may resume normal activities the day after the procedure, can start playing sports and strenuous work activities 2 weeks after the surgery.

All the patients were assessed one week after the procedure for assessment of any complication and give advice about manual lymphatic message and to stress the importance of compression garments and then 3 months and 6 months interval to assess the post operative results.

\section{Results}

The total number of procedures performed in this study were 85 , in two years from March 2009 to February 2011. There were 10 (11.8\%) male and $75(88.2 \%)$ female patient with mean age 42.4 years \pm 10.95 years.

The age range from 19 years to 67 years and the mean age 42.4 years SD 10.95 years. The mean body mass index (BMI) of the patients was $27.6 \pm 4.9$. For seven ( $8.2 \%$ ) female patients who require 2nd and/or 3rd session of VASER liposelection, the average BMI was $28.5 \pm 3.5$. In this series, the VASER Liposelection procedure was most commonly performed in the age group 41 to 50 years (chart 1).

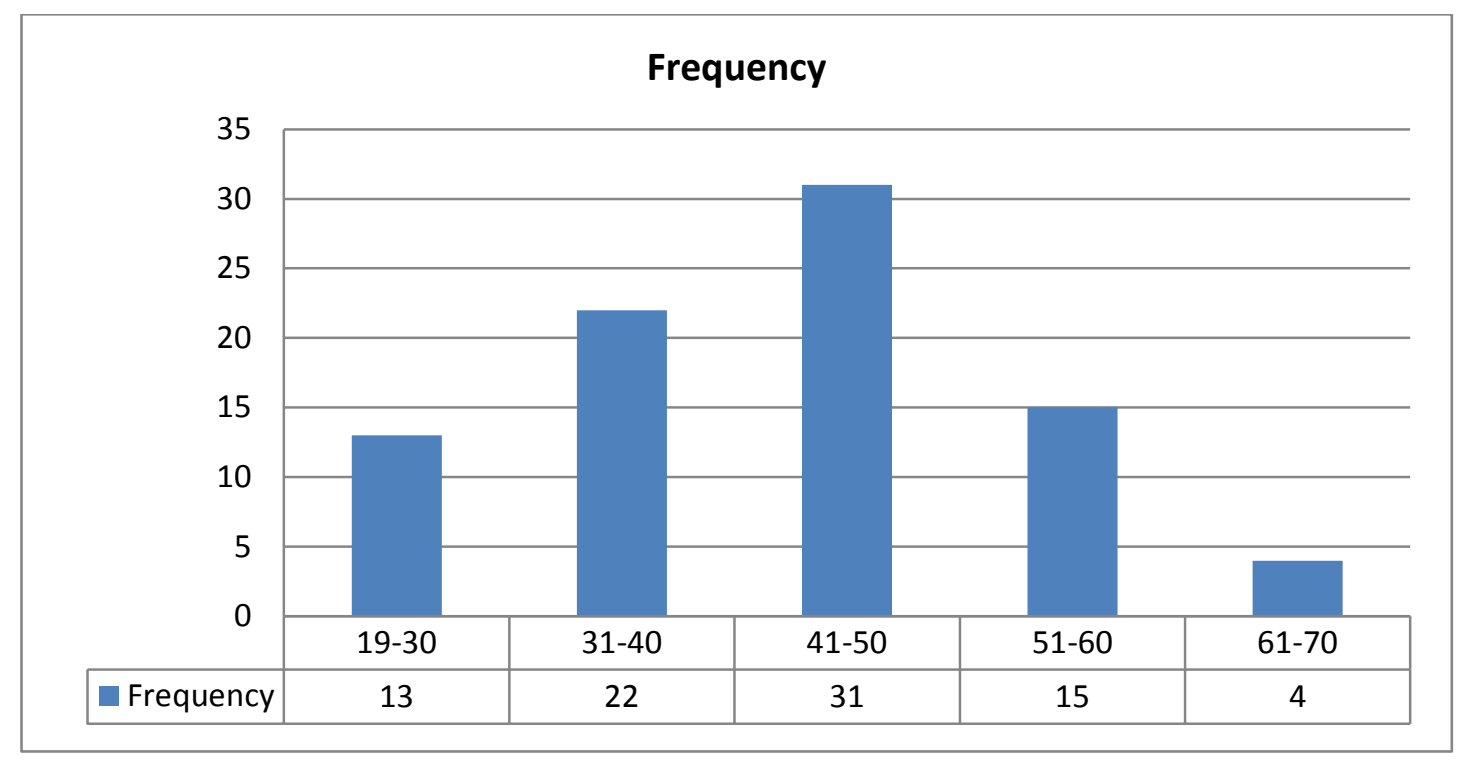

\section{Chart 1: Age Distribution}

In current series, the procedure was most commonly performed in females $88.2 \%$ as compared to males $11.8 \%$ (chart 2).

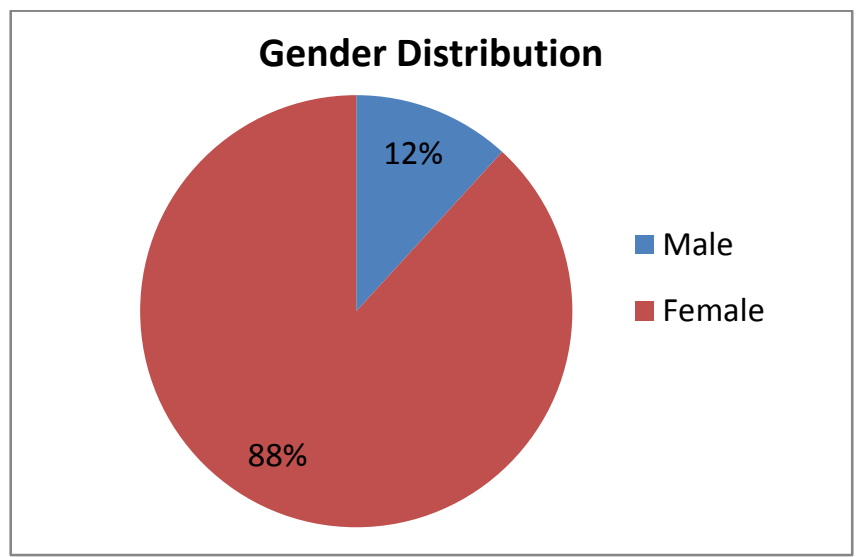

Chart 2: Gender Distribution for VASER Liposelection

All of the male patients were treated in one session of VASER liposelection and did not require 2nd or 3rd session provided BMI maintained to the pre operative level. Seven $(8.2 \%)$ female patients required 2nd session of VASER liposelection and $3(3.5 \%)$ female patients required 3rd session for complete removal of excess fat and shrinkage of the abdominal skin to achieve the satisfactory results (Table 1).

The statistical analysis of the selected group of patients who underwent for 2 nd and 3 rd session were significant ( $p$ value 0.003 ).
Photographs on the day of procedure, three months and six months after the surgery to compare and assess the post operative results were obtained. BMI is calculated and recorded pre operatively and documented on every post operative visit. No Complication were reported or observed with abdominal VASER liposelection in this study.

The client and surgeon satisfaction level achieved by using visual analogue scoring system between $75 \%$ and $95 \%$ at 3 months post operative interval and $90 \%$ to $100 \%$ at 6 months post operative interval.

\section{Discussion}

Abdominal wall consist of three layers, innermost layer is made of muscles, middle layer is made of fat and outer layer is made of skin. Huger and Nahai described the three zones of blood supply to the anterior abdominal wall ${ }^{[3,8]}$.

The suction assisted liposuction (SAL) has dramatically reduced the need for excisional body contouring techniques, especially in the abdomen. Prior to liposuction, invariably clients had to accept long abdominal scars with abdominoplasty and its related complication such as long scarring, prolong recovery, general anesthetic complication, bleeding/ haematoma, infection, deep venous thrombosis, pulmonary embolism, respiratory distress and death. Matarasso et al. analyzed the different abdominoplasty techniques and their most frequent complication. The local complication rates were $20 \%, 10.3 \%$, and $13.5 \%$ in various series. Najera et al. ${ }^{[28]}$ published a series of 200 patients, showing that the seroma rates in the abdominoplasty and lipoabdominoplasty groups 
were $16 \%$ and $31.2 \%$. Khan $\mathrm{UD}^{[26,27]}$ showing decrease incidence with progressive tension suture. The other infrequent local complication includes infection, skin necrosis, excessive scarring including hypertrophic and keloid formation, "dog ear" deformity, suture extrusion and necrosis of the umbilicus $(0.2 \%)$. Approximately $1.9 \%$ of patients suffer some degree of neurological symptoms following abdominoplasty, with the lateral femoral cutaneous nerve the most commonly involved, followed by the iliohypogastric nerve. Also the rupture of the vertical plication of the rectus abdominis sheath has been reported. The systemic complication include thromboembolism and death rate was less than $0.1 \%$ for all techniques ${ }^{[9,26,27]}$.

Reports on complication rate with abdominal liposuction in the literature from $1 \%$ to $9.3 \%{ }^{[11-21]}$. Devesh Sharma et al reported a case in 2006 of small intestinal perforation and necrotizing fasciitis after abdominal liposuction ${ }^{[22]}$. Among all the complication of liposuction, the most common complication reported is necrotising fasciitis ${ }^{[19-22]}$.

SAL has not satisfactorily addressed the needs of clients with redundant infraumbilical fat and excess skin and has produced inconsistent results in the supraumbilical area ${ }^{[10,26,27]}$. The fundamental advantage of VASER Liposelection is that fat is removed through cavitation not through mechanical avulsion as in SAL.

Patients with mild to moderate skin excess with moderate diastasis of the rectus abdominus muscles can be treated successfully in conjuction with combined procedure endoscopically or limited incision abdominoplasty. These patients would have likely required skin resection in the past. VASER liposelection is also helpful for treating the lateral flanks in patients requiring a full abdominoplasty with a long lower abdominal scar and repositioning of the umbilicus.

Various studies show the superiority of VASER Liposelection / UAL in comparison to traditional liposuction in the overall result and complication rate. Contrary to the belief, that surgery (abdominoplasty \pm SAL) is the mainstay of treatment for protuberant abdomen, VASER liposelection have revolutionised the treatment of all types of abdomen by eliminating the potential risk of surgery (Picture 1,2).

VASER liposelection helps in removing the excess fatty tissue preserving the surrounding vitals structure like blood vessels, nerves, collagen bundle etc. VASER Liposelection initiate the shrinkage of collagen fibers in the skin and collagen bundle from skin to the deeper tissues which help to shrink the skin without the need of surgical excision of lax skin (Picture 1,2). It takes approximately two to three months post operatively for skin shrinkage depending upon the degree of skin redundancy ${ }^{[25]}$.

Although, VASER Liposelection do not damage the surrounding structures like collagen bundles, nerves, blood vessels around the adipocytes, however it disrupt the lymphatic vessels resulting in lymphatic edema postoperatively. This lymphatic oedema ameliorated usually in 2-3 months with manual lymphatic message starting at 2-3 days post operatively (Picture 1\&2).

\section{Conclusion}

The results showed near complete elimination of adverse events and better overall results. Abdominal VASER liposelection is an ambulatory, day case procedure for excess fat removal and skin shrinkage with minimal or no complication performed under local anesthetic. It helps removing enormous amount of abdominal excess fat and shrinkage of lax abdominal skin avoiding the need of abdominoplasty.

\section{Compliance with ethical standards}

\section{Conflict of Interest}

None

\section{Funding}

None

\section{Ethical Approval}

Obtained

\section{Informed Consent}

Informed consent was obtained from all individual participants included in the study.

\section{Acknowledgement}

Although the study period includes two years from March 2009 to February 2011, however author have similar work practice since then and have almost the same results.

\section{References}

[1] Matarasso A (1991) Abdominolipoplasty: A system of classfication and treatment for combined abdominoplasty and suction-assisted lipectomy. Aesth Plast Surg 15(1): 111.

[2] Saldanha OR. Lipoabdominoplasty with selective and safe undermining. Aesthetic Plast Surg 2003; 22(4):3227.

[3] Huger WE (1979) The anatomic rationale for abdominal lipectomy. Am Surg 45 (9): 612-617.

[4] Alam M; Abdominal VASER Liposelection Avoiding Abdominoplasty; Biomed J Sci\&Tech Res 6(1)- 2018. BJSTR. MS.ID.001289. DOI: 10.26717/ BJSTR.2018.06.001289.

[5] Ousterhout DK. Combined suction-assisted lipectomy, surgical lipectomy, and surgical abdominoplasty. Ann Plast Surg. 1990; 24:126-32.

[6] Vidal P, Berner J E, and Will P A; Managing Complications in Abdominoplasty: A Literature Review; Arch Plast Surg. 2017 Sep; 44(5): 457-468.

[7] Zocchi M. Ultrasonic liposculpturing. Aesthetic PlastSurg 1992; 16:287-98.

[8] Nahai F, Brown GR (1976) Vasconez LO Blood supply to the abdominal wall as related to planning abdominal incisions. Am Surg 42(9): 691- 695.

[9] Matarasso A, Swift RW, Rankin M. Abdominoplasty and abdominal contour surgery: a national plastic surgery survey. Plast Reconstr Surg. 2006; 117:1797-808.

[10] Rohrich RJ, Beran JS, Kenkel JM (1998) Ultrasonic assisted liposuction. Quality medical publishing, Inc, St Louis, Missouri, US, pp. 196.

[11] Barillo DJ, Cancio LC, Kim SH, Shirani KZ, Goodwin CW (1998) Fatal and near-fatal complications of liposuction. South Med J 91:487-492.

[12] Gibbons MD, Lim RB, Carter PL (1998) Necrotizing fasciitis after tumescent liposuction. Am Surg 64:458460 . 
[13] Grazer FM, de Jong RH (2000) Fatal outcomes from liposuction: Census survey of cosmetic surgeons. Plast Reconstr Surg 105:436-446 discussion 447-448.

[14] Hanke CW, Bernstein G, Bullock S (1995) Safety of tumescent liposuction in 15,336 patients: National survey results. Dermatol Surg 21:459-462.

[15] Heitmann C, Czermak C, Germann G (2000) Rapidly fatal necrotizing fasciitis after aesthetic liposuction. Aesth Plast Surg 24:344-347.

[16] Ovrebo KK, Grong K, Vindenes H (1997) Small intestinal perforation and peritonitis after abdominal suction lipoplasty. Ann Plast Surg 38:642-644.

[17] Rao RB, Ely SF, Hoffman RS (1999) Deaths related to liposuction. N Engl J Med 340:1471-1475.

[18] Sharma D, Dalencourt G, Bitterly T, Benotti PN (2006) Small intestinal perforation and necrotizing fasciitis after abdominal liposuction. Aesth Plast Surg 30:712-716.

[19] Talmor M, Hoffman LA, Lieberman M (1997) Intestinal perforation after suction lipoplasty: A case report and review of the literature (review). Ann Plast Surg 38:169172.

[20] Toledo LS, Mauad R (2006) Complications of body sculpture: Prevention and treatment. Clin Plast Surg 33:1-11

[21] Umeda T, Ohara H, Hayashi O, Ueki M, Hata Y (2000) Toxic shock syndrome after suction lipectomy. Plast Reconstr Surg 106:204-207 discussion 208-209.
[22] Sharma D, Dalencourt G, Bitterly T, Benotti P N; Small Intestinal Perforation and Necrotizing Fasciitis After Abdominal Liposuction; Nov 2006, Aesthetic Plastic Surgery 30(6):712-6 DOI: 10.1007/s00266-006-0078-8.

[23] Klein JA; Pharmacology of lidocaine. In: Tumescent Technique: Tumescent Anesthesia and Microcanular Liposuction. St Louis, Mo: Mosby, Inc: 2000: Chap 17.

[24] Klein JA; Tumescent Liposuction and Improved Postoperative Care Using Tumescent Liposuction Garments; Dermatologic clinics; April 1995, Vol 13 (2): 329-338.

[25] Parish TD. A review: the pros and cons of tumescent anesthesia in cosmetic and reconstructive surgery. Am J Cosmet Surg. 2000;18:83-93.

[26] Khan U.D. Abdominoplasty, seroma formation and duration of hospitalisation: comparative analysis and outcome of 112 consecutive lipoabdominoplasties performed using progressive tension sutures, Eur J Plast Surg (2019), https://doi.org/10.1007/s00238-019-01531-z

[27] Khan, U.D. Risk of seroma with simultaneous liposuction and abdominoplasty and the role of progressive sutures. Aesth Plast Surg (2008) 32: 93. https://doi.org/10.1007/s00266-007-9004-y

[28] Najera RM, Asheld W, Sayeed SM, et al. Comparison of seroma formation following abdominoplasty with or without liposuction. Plast Reconstr Surg. 2011;127:41722 . 